\title{
PROPOSTA PARA IMPLANTAÇÃO DO PROGRAMA DE TRANSPLANTES INTESTINAIS NO BRASIL
}

\author{
Proposed model for a Small Bowel Transplantation Program in Brazil
}

\author{
Alexandre Bakonyi Neto
}

\begin{abstract}
RESUMO
Objetivo: 0 número de centros transplantadores que realizam transplantes de intestino tem aumentado nos últimos anos. Desde a última década, os transplantes de intestino delgado evoluíram de um procedimento experimental para uma terapêutica de preservação da vida. 0 artigo tem como objetivo descrever os critérios propostos ao M inistério da Saúde e ao Sistema Nacional de Transplantes para implantação de um programa de transplantes intestinais no B rasil. Método: 0 transplante de intestino delgado está indicado para pacientes adultos e pediátricos, com síndrome do intestino curto e falência intestinal irreversível, dependentes da Nutrição Parenteral Prolongada; pseudo obstrução, doença de microvilosidades ou tumores abdominais benignos com envolvimento do intestino delgado. Os critérios de listagem incluirão pacientes com intestino remanescente igual a ou menor de $40 \mathrm{~cm}$ aferido no momento da ressecção, perda de um acesso venoso, sem perspectivas de reassumir dietas orais sem complementação com N utrição Parenteral Prolongada, um episódio de sepse relacionado aos cateteres e disfunção hepática relacionada a N utrição Parenteral Prolongada. A perda de todos os acessos venosos, infecção não controlada, tumores malignos com acompanhamento inferior a cinco anos e doenças cardiorespiratórias sem tratamento serão considerados critérios de exclusão. A primeira etapa poderá autorizar um número menor de centros no B rasil, para aquisição de experiência. Conclusão: Concluímos que fatores relacionados ao sucesso do procedimento, como os avanços das técnicas cirúrgicas e seleção dos pacientes listados, deverão contribuir significantemente para a mel hora dos resultados.
\end{abstract}

Descritores: transplante intestinal; síndrome do intestino curto.

\section{Grau acadêmico:}

Ex-Post-Doutorate Fellow in Liver/Intestinal and Pâncreas Transplantation at University of Miami School of Medicine - Miami, EUA.

Instituição:

Prof. Assistente - Doutor do Departamento de Cirurgia e Ortopedia da Faculdade de Medicina de Botucatu - UNESP;

Chefe do Grupo de Transplantes de Fígado, Pâncreas e Intestino do Hospital das Clínicas da UNESP

Correspondência:

Alameda das Tipuanas 220, Parque das Cascatas - Botucatu - SP

CEP 18603-000

Fone: 551438142434

E-mail: fefofofo@laser.com.br

Recebido em: 06/09/2005

Aceito em: 05/10/2005

\section{INTRODUÇÃO}

Com os avanços dos cuidados intensivos neonatais e da Nutrição Parenteral Prolongada (N PP), mel hora significativa dos resultados tem sido observada em crianças e adultos submetidos a ressecções intestinais extensas. A NPP tem proporcionado sobrevidas prolongadas em pacientes com falência intestinal, muitos deles atingindo a adaptação funcional do intestino remanescente. ${ }^{1}$

Entretanto, a N PP pode predispor ao surgimento de complicações, destacando-se as relacionadas aos cateteres (infecções, tromboses dos sistemas venosos) bem como de complicações metabólicas e alterações funcionais/estruturais do fígado. Nessas situações, de grande morbidade e mortalidade, o transplante de intestino del gado surge como a única terapêutica.

Os resultados recentes têm demonstrado médias de sobrevida de $40 \%$ a $50 \%$ em cinco anos em mais de 989 pacientes adultos e pediátricos transplantados em 42 centros no exterior, com $70 \%$ a $80 \%$ dos pacientes com mais de seis meses após o transplante com função completa do enxerto e com Índice de K arnofsky de 90 a $100 \%$ em 80 a $85 \%$ dos pacientes. ${ }^{2}$ Os resultados têm mel horado nos últimos anos, tendo em vista a utilização de protocolos de imunossupressão mais específicos e eficazes; no entanto, a maior limitação ao transplante em pacientes com falência intestinal 
irreversível ainda tem sido a perda do momento ideal da indicação. As indicações do transplante na vigência de complicações irreversíveis podem inviabilizar o procedimento cirúrgico pela grande morbidade e mortalidade pós-operatória.

Em trabal ho multicêntrico realizado entre os principais centros universitários do interior do estado de São Paulo ${ }^{3}$ com 640 pacientes, avaliaram-se 251 pacientes submetidos a ressecções intestinais em graus variados. Destes pacientes, 28 evoluíram com Síndrome do Intestino Curto, com 14 óbitos decorrentes de complicações relacionadas à N PP; no entanto, apenas quatro pacientes foram encaminhados para avaliação visando um possível transplante intestinal.

Entre os fatores identificados para justificar o baixo índice de encaminhamento e avaliação, podemos destacar a excessiva expectativa na readaptação funcional intestinal em pacientes com intestino remanescente ultra-curto, bem como o desconhecimento dos recentes resultados desta modalidade de transplantes e a não regulamentação e custeio do procedimento pelo Sistema Único de Saúde no Brasil.

Por iniciativa do M inistério da Saúde e do Sistema Nacional de Transplantes, em fórum realizado em 2004, discutiu-se a necessidade de se estabelecerem critérios de caráter nacional para a inclusão de pacientes nos Cadastros Técnicos do transplante intestinal.

\section{MÉTODO:}

1 - Indicações para o Transplante Intestinal: pacientes portadores de Síndrome do Intestino Curto dependente de nutrição parenteral total permanente (N PT), pseudoobstrução I ntestinal Idiopática, doenças das M icrovilosidades ou lesões extensas da cavidade abdominal que demandem ressecções do intestino delgado.

2 - Critérios para inclusão de pacientes nos Cadastros Técnicos de Receptores de Intestino - "lista única": os pacientes deverão apresentar pelo menos um dos seguintes critérios:

Intestino delgado remanescente menor ou igual a $40 \mathrm{~cm}$, aferido no momento da ressecção intestinal, trombose de um acesso venoso profundo, incapacidade de reassumir dieta oral sem suporte da nutrição parenteral total, ter apresentado no mínimo um episódio de sepse relacionado aos cateteres da N PT, insuficiência hepática secundária a NPT e tumores benignos com comportamento localmente invasivo que demandem ressecções extensas de intestino delgado e outros órgãos abdominais (estômago; duodeno e pâncreas).

3 - Critérios de exclusão: perda completa dos acessos venosos profundos, infecções não controladas, doença neoplásica maligna com seguimento inferior a cinco anos e doenças cardiovasculares e ou pulmonares sem possibilidade de tratamento.

4 - Seleção do doador: deverão preencher os critérios de doador de múltiplos órgãos, idade de até 55 anos, índice de massa corpórea menor ou igual a 25, compatibilidade A B 0 , estabilidade hemodinâmica sem necessidade de drogas vasoativas ou em baixas doses e sorologia (IgM ) negativas para CM V e EBV.

5 - A tendimento pré-transplante: atendimento domiciliar ou internação domiciliar visando os cuidados com N PT / dieta enteral, reduzir os riscos de infecção, mel horar a qualidade de vida e maior envolvimento familiar com os cuidados e, conseqüentemente, diminuição dos custos. Deverá contemplar também a disponibilidade de cateteres implantáveis de longa permanência e os custos de internação em hospital Dia.

6 - Critérios de Urgência: pacientes com insuficiência hepática secundária a N PT, diagnosticado por meio de dosagens séricas de bilirrubinas ( $\uparrow 3 \mathrm{mg} \%$ ) e com biópsia hepática demonstrando fibrose. Para os candidatos a transplante de intestino associado ao transplante de fígado ou multivisceral, a lista de espera dos pacientes para o transplante intestinal determinará a alocação do fígado a ser transplantado concomitantemente.

7 - A tendimento pós-transplante: atendimento domiciliar ou internação domiciliar, cuidados com N PP/dietas enterais e com os cateteres implantáveis de longa permanência, internações em Hospital Dia, enteroscopias para monitoramento dos enxertos transplantados e fornecimento de drogas imunossupressoras para controle da rejeição.

\section{DISCUSSÃO}

O número de centros transplantadores autorizados e dos transplantes realizados no B rasil tem aumentado nos últi mos anos, tanto em função do aumento da demanda de pacientes, bem como de profissionais qualificados em centros de referência no exterior. Excluindo-se as diversas modalidades de transplantes de órgãos já real izados em nosso meio, os transplantes de intesti no delgado deverão ser regulamentados pel o M inistério da Saúdee pelo Sistema Nacional de Transplantes, uma vez que as propostas para a regulamentação técnica do procedimento já foram amplamente discutidas.

Os pacientes com Síndrome do Intestino Curto têm etiopatogenia multifatorial; no entanto, as indicações para a real ização do transplante intestinal são bem estabelecidas, com os resultados em longo prazo² tendo consolidado o procedimento como única terapêutica.

A demanda de pacientes com Síndrome do Intestino Curto em nosso meio não é conhecida, haja vista não existir um registro nacional que permita o cadastramento destes pacientes e, conseqüentemente, uma avaliação da real demanda para o transplante de intestino delgado, à semel hança do que ocorre em outros centros. ${ }^{4} \mathrm{Na}$ França, onde o tratamento de pacientes com falência intestinal é referendado para centros especializados no tratamento e suporte nutricional, $15 \%$ dos pacientes pediátricos em N PP desenvolvem complicações graves. ${ }^{5}$

0 transplantedeintestino delgado aindaéconsiderado um procedimento de alto risco, devendo ser reservado até o presente para pacientes com falência intestinal irreversível, sem possibilidade de readaptação ecom complicações relacionadas à alimentação parenteral.

Durante a ultima década o transplante intestinal evoluiu de um procedimento meramente experimental para uma terapia de resgate em pacientes com complicações graves, graças ao advento de avanços em técnica cirúrgica, protocolos de imunossupressão mais eficazes e, principalmente, nas indicações do procedimento em períodos que antecedem o surgimento de complicações irreversíveis. ${ }^{6,7}$

0 sucesso terapêutico nesta modalidade de transplante pode ser monitorado mundialmente pela experiência dos centros 
transplantadores, com índices de sobrevida do enxerto e do receptor após um ano, semel hantes aos de outras modalidades de transplante. A pesar da complexidade do procedimento, é o único tratamento que permite aos pacientes reassumirem a alimentação por via oral. Os resultados têm demonstrado que um enxerto funcionante pode proporcionar excelente qual idade de vida aos pacientes, ${ }^{8,9} \mathrm{com}$ menor ônus financei ro para as instituições públicas de saúde, após dois anos, quando comparado aos custos da nutrição parenteral prolongada. ${ }^{6.10}$
0 número restrito de centros de referência e da heterogeneidade das condutas no manuseio de pacientes com falênciaintestinal em nosso meio torna fundamental o estabelecimento de critérios de caráter nacional para a inclusão de pacientes nos Cadastros Técnicos de Receptores de Intestino - "lista única" e do Regulamento Técnico que dispõe sobre as atividades detransplante. 0 consenso do último Fórum foi a adequação de alguns critérios para o nosso meio, visando principal mente 0 acesso mais precoce dos pacientes para o transplante e, conseqüentemente, diminuir a morbidade e mortalidade pós-operatória. ${ }^{11}$

\section{ABSTRACT}

Objective: The amount of transplantation centers performing intestinal transplants have increased in the last few years. O ver the past decade, small bowel transplantation (SBT) has developed from an experimental procedure to a life-saving therapy. Herein, it will be described the present policy proposed to the B razilian Health M inistry, in order to start-up a intestinal transplant program in B razil. Methods: SBT is indicated for adult and pediatric patients with short bowel syndrome and those dependent to Prolonged Parenteral Nutrition (PPN), irreversible intestinal failure, pseudoobstruction, microvilous disease or benign abdominal tumors with involvement of the small bowel. The list criteria include patients with remaining small bowel less/equal to $40 \mathrm{~cm}$ measured at the resection time; loss of a venous access; no possibility to resume oral intake without PPN support; one episode of catheter-related sepsis and PPN-related liver dysfunction. L oss of all venous accesses, uncontrolled infections, mal ignant tumors with less than 5 years follow-up and no-treated cardiovascular/lung diseases were the excluding criteria. A first step may only certify a lower amount of reference centers all over the country, aiming to better develop the expertise. Condusion: It was concluded that factors related to a successful procedure, such as advancements in the surgical technique and the selection of patients on lists must significantly contribute to the improvement of outcomes.

Keywords: intestinal transplantation; short bowel syndrome.

\section{REFERÊNCIAS}

1. Azuma T, Nakai H, Fukuzawa M. Potential candidates for small bowel transplantation: from our experience and survey of home parenteral nutrition in Japan. Transplant Proc 1998;30:2529.

2. Grant D. Intestinal transplantation: 2005 report of the International Registry (Presented at IX International Small Bowel transplant Symposium). BruxelasBelgica, 2005.

3. Bakonyi Neto, A, Takegawa B, Ortolan E et al. Demographic of Short Gut Syndrome: Increasing demand is not followed by referral of potential candidates for small bowel transplantation. Transplant Proc 2004; 36(2): 259-260.

4. Kurlberg G, Forssell H. National registry of patients with short bowel syndrome (Presented at VIII International Small Bowel Transplant Symposium). MiamiFlorida, 2003

5. Ricour C, Gorski AM, Goulet O. Clin Nutr 1990;9:65.
6. Abu-Elmagd KM, Reyes J, Fung JJ. Evolution of clinical intestinal transplantation: improved outcome and cost-effectiveness. Transplant Proc 1999;31 (1-2),582-84.

7. American Gastroenterological Association medical position statement: short bowel syndrome and intestinal transplantation. Gastroenterology 2003;124(4),1105-10.

8. DiMartini A, Rovera G, Grahan T. Quality of life after small bowel transplantation and among home parenteral nutrition patients. Journal Parenteral and Enteral Nutrition 1998;22(6), 362-67.

9. Sudan D, Iyer K, Horslen S. Assessment of quality of life after pediatric intestinal transplantation by parents and pediatric recipients using the child health questionnaire. Transplant Proc 2002; 34(3),963-964.

10. Schalamon J, Mayr JM, Hollwarth ME. Mortality and economics in short bowel syndrome. Best Pract Res Clin Gastroenterol 2003;17(6),931-942.

11. Fecteau A, Alkinson P, Grant D. Early referral is essential for successful pediatric small bowel transplantation. The Canadian experience. J Pediatric Surg 2001; 36(5),681-84. 